ISPRS International

Journal of

Geo-Information

ISSN 2220-9964

www.mdpi.com/journal/ijgi

Article

\title{
EASE-Grid 2.0: Incremental but Significant Improvements for Earth-Gridded Data Sets
}

\author{
Mary J. Brodzik *, Brendan Billingsley, Terry Haran, Bruce Raup and Matthew H. Savoie \\ National Snow \& Ice Data Center, Cooperative Institute of Environmental Sciences, University of \\ Colorado, 449 UCB, Boulder, CO 80309, USA; E-Mails: brendan.billingsley@nsidc.org (B.B.); \\ tharan@nsidc.org (T.H.); braup@nsidc.org (B.R.); savoie@nsidc.org (M.H.S.) \\ * Author to whom correspondence should be addressed; E-Mail: brodzik@ nsidc.org; \\ Tel.: +1-303-492-8263; Fax: +1-303-492-2468.
}

Received: 30 January 2012; in revised form: 28 February 2012 / Accepted: 1 March 2012 /

Published: 13 March 2012

\begin{abstract}
Defined in the early 1990s for use with gridded satellite passive microwave data, the Equal-Area Scalable Earth Grid (EASE-Grid) was quickly adopted and used for distribution of a variety of satellite and in situ data sets. Conceptually easy to understand, EASE-Grid suffers from limitations that make it impossible to format in the widely popular GeoTIFF convention without reprojection. Importing EASE-Grid data into standard mapping software packages is nontrivial and error-prone. This article defines a standard for an improved EASE-Grid 2.0 definition, addressing how the changes rectify issues with the original grid definition. Data distributed using the EASE-Grid 2.0 standard will be easier for users to import into standard software packages and will minimize common reprojection errors that users had encountered with the original EASE-Grid definition.
\end{abstract}

Keywords: EASE-Grid 2.0; EASE-Grid; WGS 84; equal-area projections; Earth-gridded

\section{EASE-Grid History and Attributes}

Defined in the early 1990s as part of the NOAA/NASA Polar Pathfinder Program for gridded, satellite-derived passive microwave brightness temperatures, the Equal-Area SSM/I Earth Grid (EASE-Grid) was simple to use and understand. The original definition [1,2] specified three 
projections together with the Backus-Gilbert interpolation method used for the SSM/I (Special Sensor Microwave/Imager) Pathfinder data set [3]. The EASE-Grid projection and gridding scheme was quickly adopted and used with different interpolation methods by the SMMR, AVHRR and TOVS Polar Pathfinder projects, and has been used in the production of numerous other data sets since then. Over time, the authors realized that the projection and gridding scheme had become so widely used that the $\mathrm{SSM} / \mathrm{I}$ in the original name was a misnomer, since there was nothing SSM/I-specific about the projection and grid definitions. By 2002, Brodzik and Knowles [4] decided to retain the acronym but changed the meaning to "Equal-Area Scalable Earth-Grid", to emphasize the versatility of applications it enjoyed. Today, the term EASE-Grid refers to the three original projections and associated gridding scheme, but does not include prescriptions for binning or interpolation methods.

The EASE-Grid definition [4] specifies a set of three equal-area projections together with an infinite set of potential grid (spatial resolution and coverage) definitions. The EASE-Grid projections comprise polar aspect Lambert azimuthal equal-area projections (Figure 1) for Northern or Southern Hemisphere, and a cylindrical equal-area projection with standard parallels at $\pm 30^{\circ}$ (Figure 2) for applications in midand low-latitude regions.

Figure 1. Northern and Southern EASE-Grid projections. See second figure in Section 2 for zoomed corner subset.
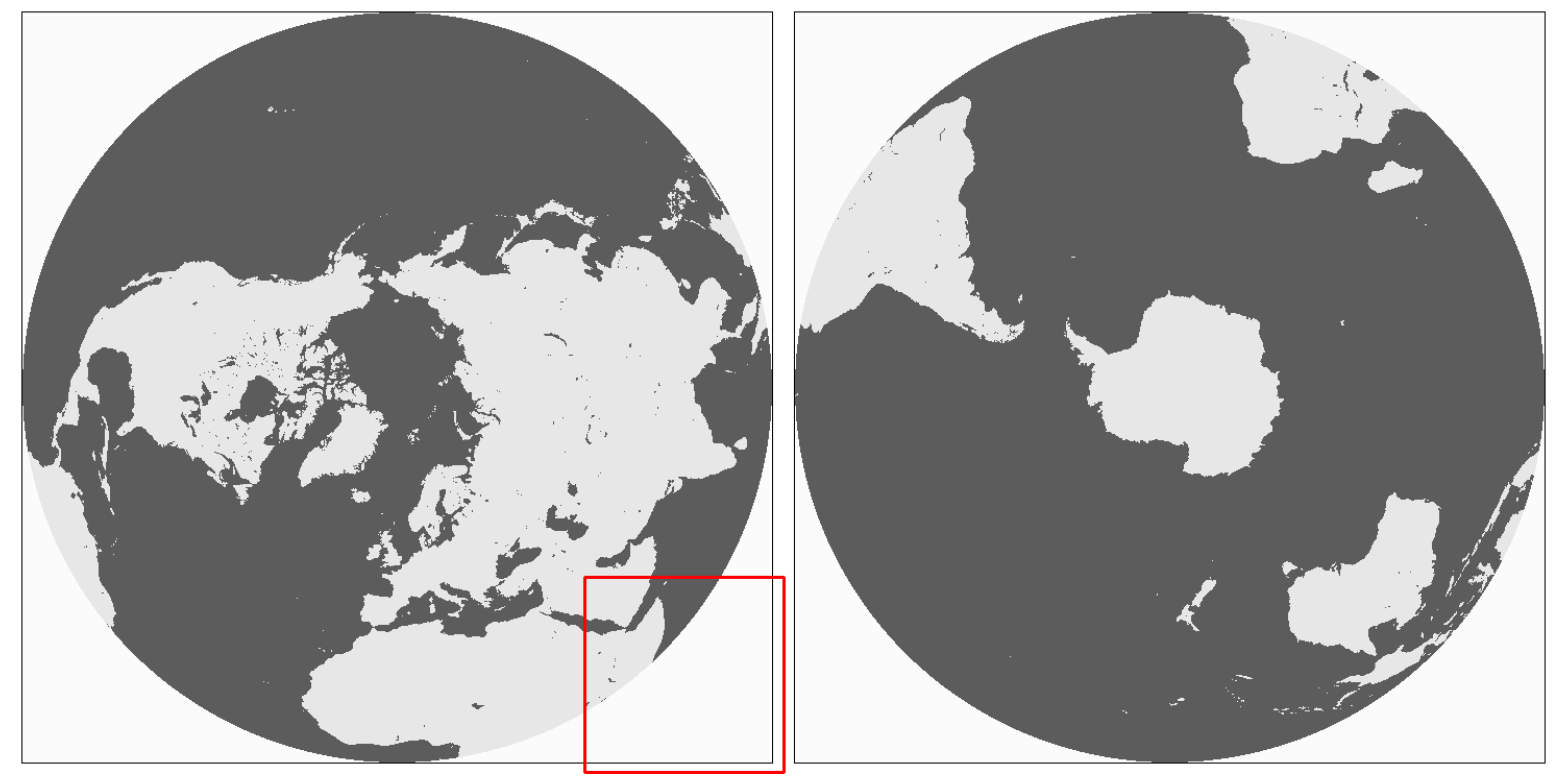

The first EASE-Grids, defined for SSM/I gridded brightness temperatures at 25-km resolution, spanned the full Northern and Southern Hemispheres in the azimuthal aspects and the global projection to a latitude of $\pm 86.72^{\circ}$. This poleward extent was inside the data area near the pole for SSM/I, where no data were collected by the sensor due to orbital inclination and the sensor geometry. The grid resolution was chosen to approximate the SSM/I spatial sampling resolution of $25 \mathrm{~km}$. The SSM/I sensor also included one scanning frequency at $85 \mathrm{GHz}$ that was sampled at twice the spatial resolution of the remaining frequencies. Higher-resolution, 12.5-km EASE-Grids were also defined for each projection with the center location of every other $12.5-\mathrm{km}$ cell co-located with the center of a $25-\mathrm{km}$ cell. 
A number of data set producers (including [5-10]) have adopted the EASE-Grid format. For equal-area projections, area calculations are accomplished by simply multiplying a constant cell area by the sum of grid cells with particular characteristics. For polar applications, this simple operation is much easier than area calculations on the common alternative polar stereographic grids, where grid cell area is a function of latitude. Data set producers also take advantage of the EASE-Grid "scalability" feature by defining custom grid resolutions and areal coverages to suit specific applications [11].

Figure 2. Cylindrical EASE-Grid projection.

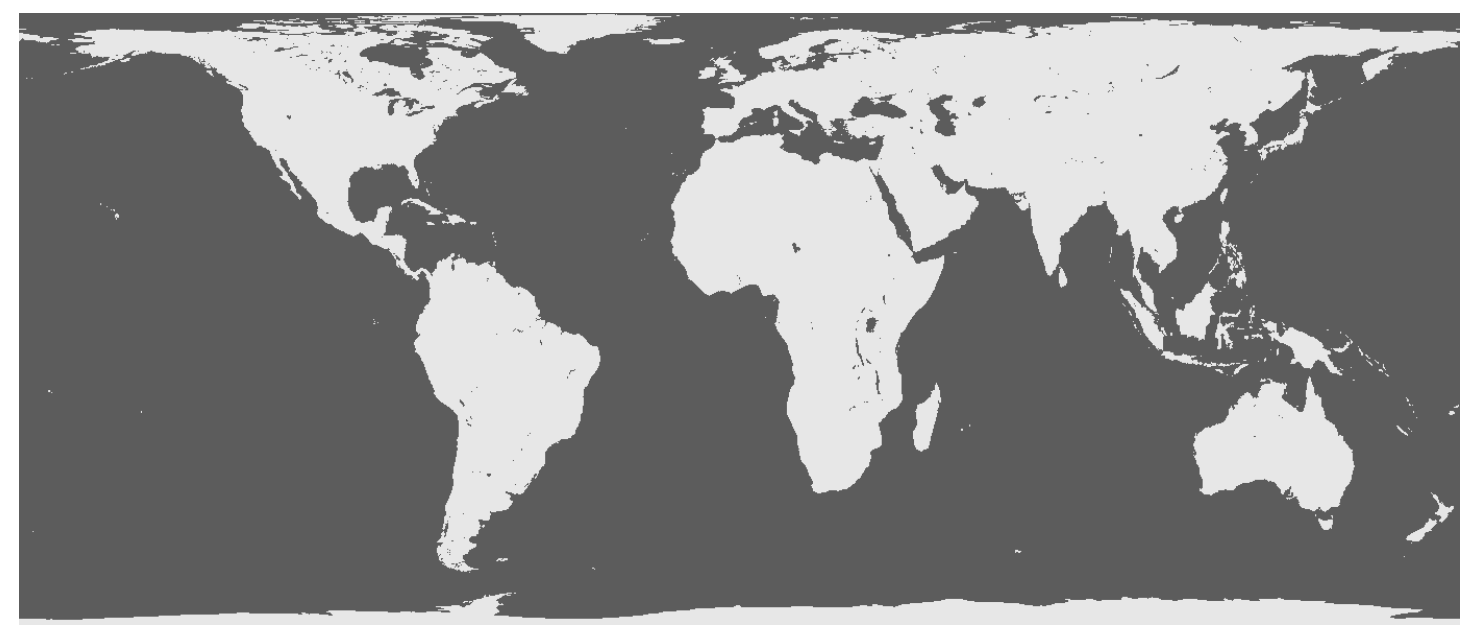

In the years since the original EASE-Grid definition, other authors have recommended the use of equal-area projections for gridded data sets. The Peters projection (a cylindrical equal-area projection like the one chosen for the cylindrical EASE-Grid, but with standard parallels at $\pm 45^{\circ}$ instead of $\pm 30^{\circ}$ ) was recommended for ocean-scale oceanographic applications by Krause and Tomczak [12], who sought the qualities of fidelity of area combined with a rectangular coordinate system. Trischenko et al. [13] have adopted a Lambert azimuthal equal-area projection for their MODIS Arctic Circumpolar Mosaic, noting that this choice of projection reduces image distortion and preserves image information content, which they consider an advantage over gridded data in the typical "lat-lon" (Plate-Carrée, or equidistant cylindrical) projection or the MODIS sinusoidal projection.

The original EASE-Grid was defined as an easier alternative to swath format data in order to support standardized spatial comparisons of geophysical phenomena derived from satellite microwave data [1]. Over time, EASE-Grid has been adopted and used by broader communities to produce gridded data sets that include satellite passive microwave brightness temperatures $[3,8,14,15]$, permafrost land classifications [5,16], atmospheric parameters [6], surface reflectance, albedo and skin temperature [7], snow and ice products [10,17-20] and soil moisture [9]. Judging by the variety and number of these data set producers, EASE-Grid satisfies a need in communities that choose equal-area projections for gridded data sets.

\section{EASE-Grid Limitations}

EASE-Grid is a popular gridding choice for satellite-derived data sets, especially applications representing geophysical parameters in the polar regions. However, while the original EASE-Grid is 
easy to learn and easy to adapt to new applications, it suffers from a set of limitations that make its use with current software packages error-prone for users, mapping experts and non-experts alike.

In the following discussion, we use the ISO19111 terminology explained in Section 2.1.2 and Appendix A of Iliffe and Lott [21], with some additional notes relevant to issues with the EASE-Grid definition indicated in italics.

An ellipsoid is a closed surface formed by the rotation of an ellipse about its shorter (minor) axis.

A coordinate system (CS) is a set of rules to define how coordinates are assigned to points, usually by means of associated axes.

A coordinate reference system (CRS) is a CS that defines position, scale and orientation of its axes defined with respect to an object, which for our purposes is the Earth.

Geodetic latitude is the angle from the equatorial plane to the perpendicular to the ellipsoid through a given point.

Geodetic longitude is the angle from the prime meridian plane to the meridian plane of a given point.

An ellipsoidal coordinate system (ECS) is a CS specified by geodetic latitude and longitude.

A geodetic datum defines the relationship of a 2- or 3-dimensional CS to the Earth. A geodetic datum defines the position of the origin, the scale, and the orientation of the axes of an ECS with respect to a particular Earth ellipsoid. The characteristics of several Earth ellipsoids are included in Table 1.

A geodetic coordinate reference system (GCRS) is an ECS defined for a particular geodetic datum.

A coordinate conversion is a change of coordinates from one CRS to another, in which the CRSs are either based on the same datum or, if they are based on different datums, no algorithm has been applied to transform the coordinates from one datum to the other.

A coordinate transformation is a change of coordinates from one CRS to another in which the CRSs are based on different datums. In this case, a coordinate transformation algorithm is applied to convert the coordinates of one CRS to conform to the datum of the other CRS.

A map projection is a coordinate conversion from an ECS to a plane. A map projection is defined by various projection parameters, including the projection ellipsoid, the ECS coordinates of the projection origin in the plane, the orientation of the projected axes in the plane with respect to the ECS, and other projection-specific parameters.

A projected coordinate reference system (PCRS) is a CRS derived from a GCRS by applying a specified map projection. Note that the projection ellipsoid specified in the map projection may or may not match the datum specified in the GCRS.

The original EASE-Grid map projections were defined with a spherical Earth model for the projection ellipsoid, namely the International 1924 Authalic Sphere (see Table 1 and Appendix B). However, most of the data currently stored in EASE-Grid are derived from satellite-based sensors, which have WGS 84 as the GCRS datum. Iliffe and Lott [21] make a distinction between conversions and transformations by pointing out that conversions are defined and are considered to be exact. They point out that coordinate conversions therefore result in no loss of positional accuracy, which was also explained in Brodzik and Knowles [4] to justify the choice of a spherical Earth model in the original EASE-Grid map projection definitions.

The decision to use a spherical Earth model for the PCRS projection ellipsoid with data that are referenced to a GCRS datum for a different ellipsoid does not result in loss of positional accuracy 
during coordinate conversion, as long as software packages consider the PCRS map projection ellipsoid and the GCRS reference datum separately. The distinction must be handled correctly by any software that reprojects EASE-Grid data: this requires that users understand the software well enough to know when to apply coordinate conversions alone (reprojecting with no change in GCRS reference datum) versus coordinate transformations (reprojecting and changing the GCRS reference datum). EASE-Grid data users can encounter problems because a number of popular software packages, including ArcGIS, make the assumption that the PCRS map projection ellipsoid and the GCRS reference datum are the same. These applications typically provide no way to specify different values, or suggest applying an inappropriate coordinate transformation instead of the appropriate coordinate conversion [22]. Without a thorough understanding of how the software is handling conversions and transformations, an unsuspecting user may perform a reprojection of data from EASE-Grid that erroneously assumes the GCRS reference datum is the 1924 Authalic Sphere, rather than the actual GCRS reference datum, which is the WGS 84 datum.

Table 1. Characteristics of Earth ellipsoids (Table 2.1, p. 10 in [21] and Appendix B) discussed in the text.

\begin{tabular}{|l|l|l|l|l|}
\hline \multicolumn{5}{|c|}{ Earth Ellipsoids } \\
\hline Name & $\begin{array}{l}\text { Equatorial } \\
\text { Radius } \\
(\mathbf{m})\end{array}$ & Flattening & $\begin{array}{l}\text { Calculated Polar } \\
\text { Radius }\end{array}$ & $\begin{array}{l}\text { Calculated } \\
\text { Eccentricity }\end{array}$ \\
\hline $\begin{array}{l}\mid c \\
\text { 1924 Ellipsoid }\end{array}$ & 6378388 & $1 / 297.0$ & $b=a(1-f)$ & $e=\sqrt{2 f-f^{2}}$ \\
\hline $\begin{array}{l}\text { International } \\
\text { 1924 Authalic } \\
\text { Sphere }\end{array}$ & 6371228 & 0 & 6356911.946 & 0.0819918899790 \\
\hline WGS 84 & 6378137 & $1 / 298.257223563$ & 6356752.314 & 0.0818191908426 \\
\hline
\end{tabular}

In the case of the coarser resolution EASE-Grids (12.5-km or coarser), the consequences of these errors were not drastic (on the order of one or two grid cell locations). However, recent applications of EASE-Grid, for example, to store data at 500-m resolution, have led to significant errors in reprojection as in Figure 3.

Furthermore, formats do not always allow for different GCRS reference datums and PCRS projection ellipsoids. The popular GeoTIFF file format standard requires that the PCRS projection ellipsoid be the same as the GCRS reference datum [23]. EASE-Grid data cannot be legitimately distributed in GeoTIFF format, therefore, without first applying a coordinate conversion (reprojection with no change in GCRS reference datum) to change the projection ellipsoid from the authalic sphere to WGS 84 [22,24]. An alternative but awkward approach would be to apply a coordinate transformation to perform the datum 
shift from WGS 84 to the authalic sphere, but this would change latitude values by as much as 0.19 degrees, or $20 \mathrm{~km}$ near $\pm 45^{\circ}$ [25]. For either of these alternatives, the resulting data would be a legitimate GeoTIFF file, but could no longer be designated EASE-Grid.

Figure 3. Example of NASA Operation IceBridge flight tracks overlaid on NASA Blue Marble basemap of central eastern Greenland. Blue Marble data are produced in a Plate-Carrée projection referenced to the WGS 84 datum and stored as WMS layers in the original EASE-Grid. The left-hand base map was derived by coordinate transformation into the original EASE-Grid, with an inappropriate datum shift. The right-hand base map was derived correctly by coordinate conversion only (reprojection without the datum shift). The error in the basemap was not identified until the flight manager notified us that the flight track actually traversed down the middle of Daugaard-Jensen Gletscher in the upper Scoresby Sund fjord (top center circle in each image) and that the flightlines parallel to Blosseville Kyst (lower two circles in each image) were performed over sea ice along the coast, not inland.
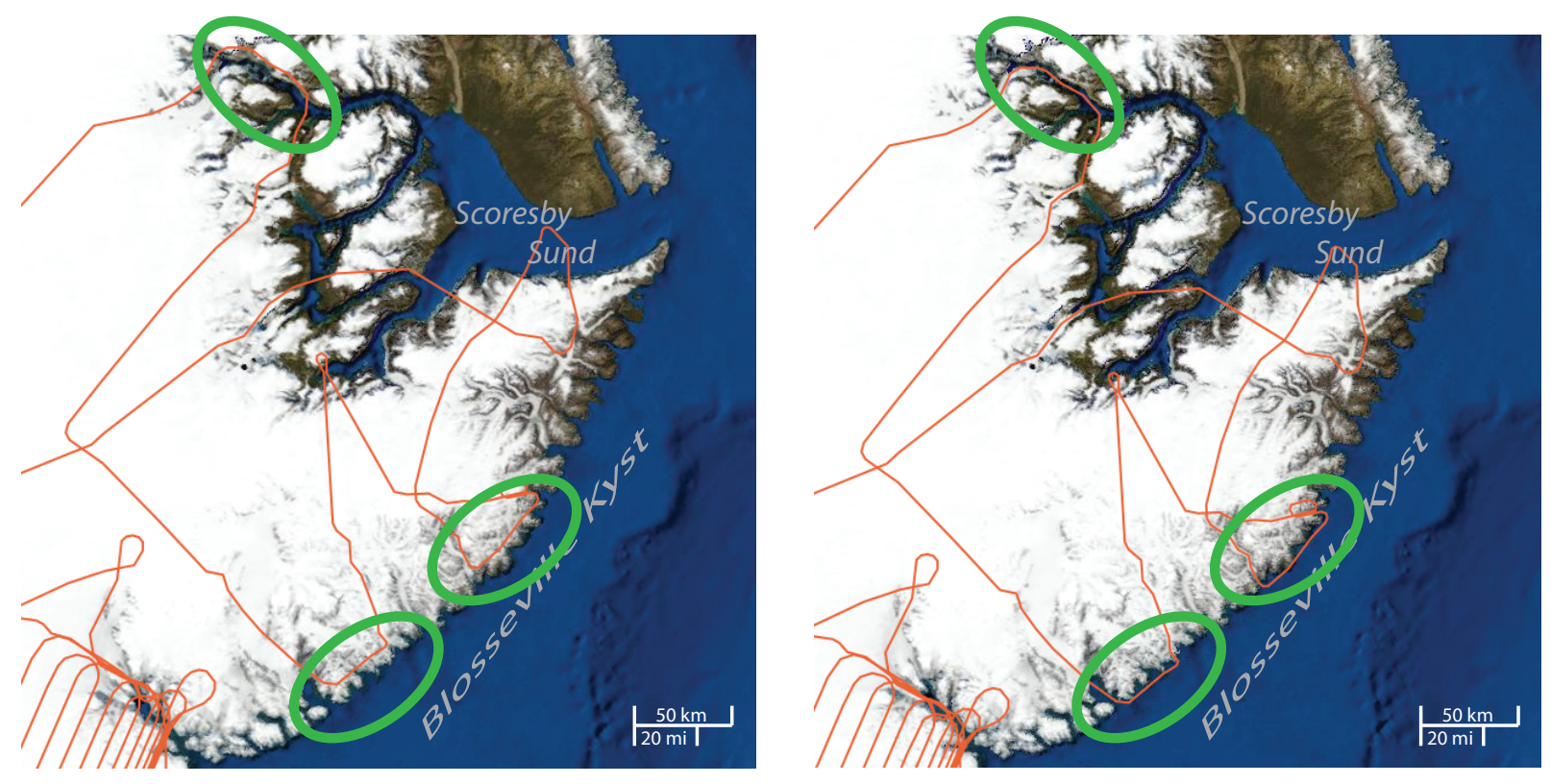

Finally, idiosyncracies at the corners and edges of grids were introduced in the azimuthal EASE-Grids, due to grid definitions with an odd number of pixels, with the pole located at the center of the center grid cell. Grids at multiples of the original $25-\mathrm{km}$ spatial resolution were defined in a bore-centered fashion. The equator was properly contained (in the mathematical sense of a proper subset) within the extent of the enclosing rectangular grid extent. These choices caused two problems. First, the spatial coverage near each of the four corners of the azimuthal projections extends beyond the opposite pole. While projection coordinates in meters from the projection original are defined at the corners of these grids, the geographic (latitude, longitude) coordinates are undefined (literally "off the Earth") at locations near the corners of the grid (Figure 4). Second, the bore-centered relationship forced a partial-cell-sized offset in spatial coverage, so related grids always had different spatial extents. The original justification for the bore-centered relationship was to achieve computational speed in sampling from a finer- to a coarser-resolution grid: no averaging would be required, since each coarse resolution cell was at every 
other fine-resolution cell location. Over time, this decision has proven difficult to justify. Most users find nested grid cells, that is, four $12.5-\mathrm{km}$ cells nested in a single $25-\mathrm{km}$ cell, easier to conceptualize. Users also consider that grid subsampling rather than averaging is inappropriately discarding data.

Figure 4. Zoomed area of lower right corner of Northern EASE-Grid projection in Figure 1, indicating 25-kilometer corner pixels with undefined (latitude, longitude) coordinates. All corners are symmetric.

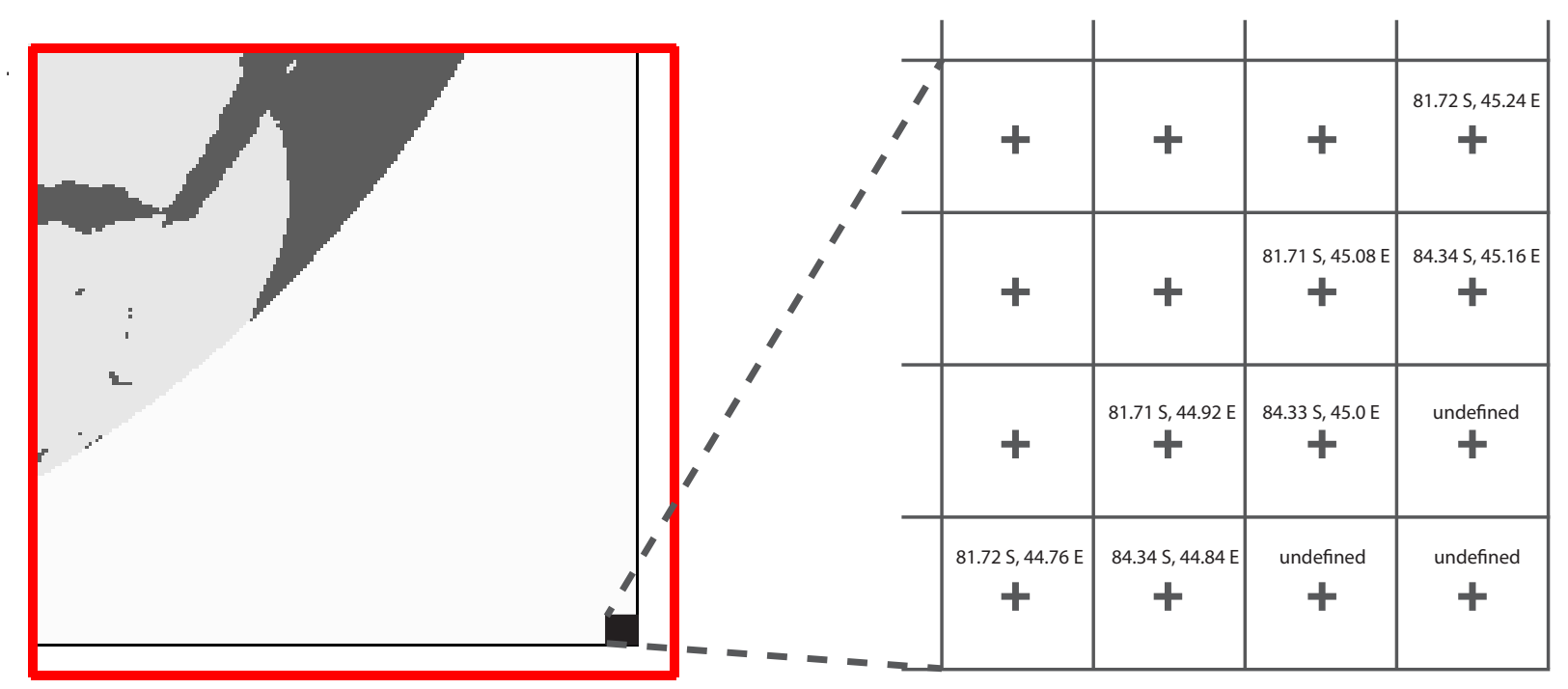

While the nominal cell size of the $25 \mathrm{~km}$ grids was $25 \mathrm{~km} \times 25 \mathrm{~km}$, the actual cell dimension was slightly larger, $25.06725 \mathrm{~km} \times 25.06725 \mathrm{~km}$, to make the global grid exactly span the equator. Once the scale was chosen for the cylindrical grid, the same scale was used for the azimuthal grids. This decision made documentation simple, with the same scale for all three projections. However, there is no mathematical justification for this decision.

\section{EASE-Grid 2.0 Definition}

We propose the following new EASE-Grid 2.0 definition to achieve the following goals: (1) make the GCRS reference datum and PCRS projection ellipsoid the same so that formats like GeoTIFF can easily accommodate the data and software packages can easily and properly reproject the data, (2) make nested grid definitions simpler, (3) eliminate undefined geographic coordinates at corners of azimuthal grids (4) decouple scales between cylindrical and azimuthal grids, and (5) select new dimensions that will immediately distinguish data in the EASE-Grid 2.0 format from the original EASE-Grid format.

The EASE-Grid 2.0 definition comprises the same three equal-area projections defined in the original, except that the PCRS projection ellipsoid is WGS 84 rather than the International 1924 Authalic Sphere. Data referenced to the WGS 84 datum and stored in EASE-Grid 2.0 projections can now be formatted directly as GeoTIFF without any reprojection. The likelihood that common software packages will correctly handle reprojection is much higher than with EASE-Grid data.

Numbers of rows and columns of the azimuthal grids are even, with the respective pole on the azimuthal grids located at the intersection of the center four grid cells (Figure 5). 
Figure 5. Relative gridding schemes for representative azimuthal $25 \mathrm{~km}$ and $12.5 \mathrm{~km}$ original EASE-Grid ((Left), bore-centered) vs. EASE-Grid 2.0 ((Right), nested) cells near the pole.
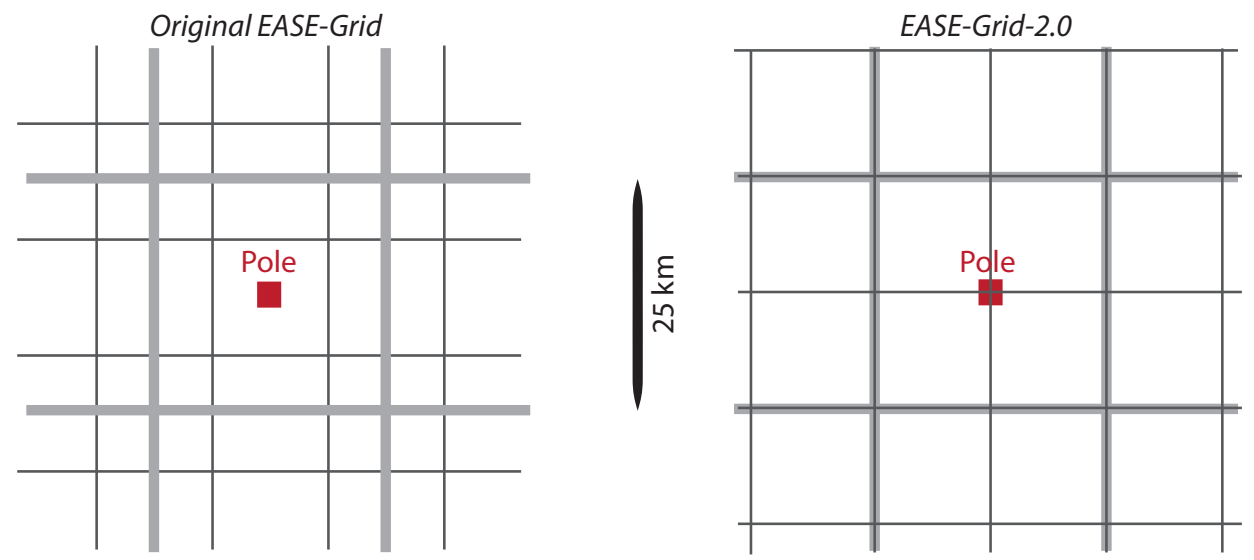

The scale of the cylindrical grid will again be determined as the closest value to the desired nominal scale such that an exact number of cells spans the equator; however, corresponding azimuthal grid scales will be exact. For example, the new $25 \mathrm{~km}$ cylindrical grid cell size is $25,025.2600081 \mathrm{~m}$, but the new $25 \mathrm{~km}$ azimuthal grid cell size is exactly $25,000.0 \mathrm{~m}$.

Figure 6. Relationship of equator to right edge of grid coverage in Northern Hemisphere azimuthal 25-kilometer original EASE-Grid ((Left), equator enclosed within spatial coverage) vs. EASE-Grid 2.0 ((Right), equator slightly outside spatial coverage). Southern Hemisphere grids are defined likewise. Curvature of equator is greatly exaggerated relative to edge of grid.
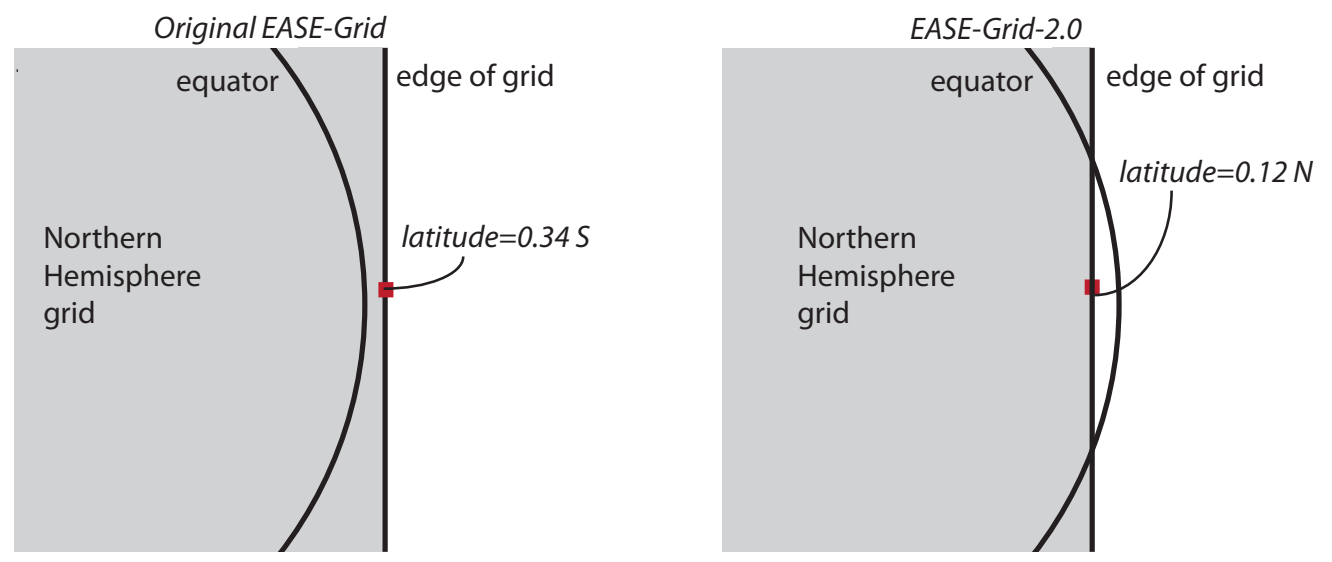

With the exact scale of $25 \mathrm{~km}$, we chose the number of cells in the new $25 \mathrm{~km}$ azimuthal grids to be 720 columns by 720 rows. This positions the equator slightly outside the grid coverage at each of the four array edges (Figure 6), with a small sector of the hemisphere outside the spatial coverage of the grid. We chose this dimension (1) to avoid the original problem with undefined latitude/longitude values in the grid corners, (2) to promote the many exact multiples and divisors for nested and nesting grids that are possible with the value 720, and (3) to indicate by different grid dimensions alone that the new grids are different from the original EASE-Grids (the original $25 \mathrm{~km}$ azimuthal EASE-Grid dimensions are 721 columns $\times 721$ rows). 


\section{Conclusions}

The proposed definition of EASE-Grid 2.0 represents relatively small but significant changes that will allow for easier data distribution in contemporary formats like GeoTIFF and will prevent certain avoidable software reprojection issues. We expect that nested grid definitions will be easier for users to understand; defining nested rather than bore-centered relationships will allow grid definitions at multiple scales to share exact spatial coverage. Decoupling cell sizes between cylindrical and azimuthal grids will make it easier to document and use the azimuthal grids. Finally, the choice of slightly different grid dimensions and the new scales eliminates undefined grid coordinates at the azimuthal grid corners, while providing the user an easily recognizable clue (different dimensions) to identify the grid as EASE-Grid 2.0.

Two major NASA-funded projects, SMAP (Soil-Moisture Active-Passive) and the MEaSUREs (Making Earth Science Data Records for Use in Research Environments) cryosphere grant led by D. Robinson have currently adopted EASE-Grid 2.0 as the standard format for gridded data sets, including SMAP soil moisture and freeze/thaw data and MEaSUREs Northern Hemisphere terrestrial, sea ice and Greenland ice sheet snow data. We recommend that producers of new data sets use the new EASE-Grid 2.0 standard to take advantage of the incremental improvements defined here. We also recommend that any reprocessing that is performed on legacy EASE-Grid data sets also make the change to EASE-Grid 2.0.

\section{Acknowledgements}

The authors wish to thank R. Armstrong, D. Scott, R. Weaver and three anonymous reviewers for comments that improved this manuscript.

\section{References}

1. Armstrong, R.L.; Brodzik, M.J. An Earth-Gridded SSM/I Data Set for Cryospheric Studies and Global Change Monitoring. Adv. Space Res. 1995, 16, 155-163.

2. Armstrong, R.L.; Brodzik, M.J.; Varani, A. The NSIDC EASE-Grid: Addressing the Need for a Common, Flexible, Mapping and Gridding Scheme. Earth System Monit. 1997, 7, 6-7.

3. Armstrong, R.L.; Knowles, K.W.; Brodzik, M.J.; Hardman, M.A. DMSP SSM/I-SSMIS Pathfinder Daily EASE-Grid Brightness Temperatures; Digital Media; National Snow and Ice Data Center: Boulder, CO, USA, 1994; updated 2012.

4. Brodzik, M.J.; Knowles, K.W. EASE-Grid: A Versatile Set of Equal-Area Projections and Grids. In Discrete Global Grids; Goodchild, M., Ed.; National Center for Geographic Information and Analysis: Santa Barbara, CA, USA, 2002. Available online: http://www.ncgia.ucsb.edu/ globalgrids-book/ease_grid/ (accessed on 27 November 2011).

5. Brown, J.; Ferrians, O.J., Jr.; Heginbottom, J.A.; Melnikov, E.S. Circum-Arctic Map of Permafrost and Ground Ice Conditions; Digital Media; National Snow and Ice Data Center/World Data Center for Glaciology: Boulder, CO, USA, 1998; revised February 2001. 
6. Francis, J.; Schweiger, A. TOVS Pathfinder Path-P Daily Arctic Gridded Atmospheric Parameters; Digital Media; National Snow and Ice Data Center: Boulder, CO, USA, 1999; updated 2008.

7. Fowler, C.; Maslanik, J.; Haran, T.; Scambos, T.; Key, J.; Emery, W. AVHRR Polar Pathfinder Twice-Daily $5 \mathrm{~km}$ EASE-Grid Composites V003; Digital Media; National Snow and Ice Data Center: Boulder, CO, USA, 2000; updated 2007.

8. Knowles, K.W.; Njoku, E.G.; Armstrong, R.L.; Brodzik, M.J. Nimbus-7 SMMR Pathfinder Daily EASE-Grid Brightness Temperatures; Digital Media; National Snow and Ice Data Center: Boulder, CO, USA, 1999.

9. Njoku, E. AMSR-E/Aqua Daily L3 Surface Soil Moisture, Interpretive Parameters, and QC EASE-Grids V002; Digital Media; National Snow and Ice Data Center, Boulder, CO USA, 2008, updated daily.

10. Arctic and Antarctic Research Institute. Sea Ice Charts of the Russian Arctic in Gridded Format 1933-2006; Smolyanitsky, V., Borodachev, V., Mahoney, A., Fetterer, F., Barry, R., Eds.; Digital Media; National Snow and Ice Data Center: Boulder, CO, USA, 2007.

11. Kim, E.J.; O’Kray, C.; Hinds, N.; England, A.W.; Brodzik, M.J.; Knowles, K.; Hardman, M. A Custom EASE-Grid SSM/I Processing System. In Proceedings IGARSS '98, Seattle, WA, USA, 6-10 July 1998; pp. 1112-1114.

12. Krause, G.; Tomczak, M. Do Marine Scientists Have a Scientific View of the Earth? Oceanography 1995, 8, 11-16.

13. Trishchenko, A.P.; Luo, Y.; Khlopenkov, K.V.; Park, W.M.; Wang, S. Arctic Circumpolar Mosaic at $250 \mathrm{~m}$ Spatial Resolution for IPY by Fusion of MODIS/TERRA Land Bands B1-B7. Int. J. Remote Sens. 2009, 30, 1635-1641.

14. Knowles, K.W.; Savoie, M.H.; Armstrong, R.L.; Brodzik, M.J. AMSR-E/Aqua Daily EASE-Grid Brightness Temperatures; Digital Media; National Snow and Ice Data Center: Boulder, CO, USA, 2006; updated 2011.

15. Long, D.; Stroeve, J. Enhanced Resolution SSM/I and AMSR-E Daily Polar Brightness Temperatures; Digital Media; National Snow and Ice Data Center: Boulder, CO, USA, 2011.

16. Qiu, G.; Zhou, Y.; Guo, D.; Wang, Y. Maps of Geocryological Regions and Classifications; Digital Media; National Snow and Ice Data Center/World Data Center for Glaciology: Boulder, CO, USA, 2002.

17. Armstrong, R.L.; Brodzik, M.J. Northern Hemisphere EASE-Grid Weekly Snow Cover and Sea Ice Extent Version 3; Digital Media; National Snow and Ice Data Center: Boulder, CO, USA, 2005; updated 2007.

18. Hall, D.K.; Riggs, G.A.; Salomonson, V.V. MODIS/Terra Sea Ice Extent Daily L3 Global $1 \mathrm{~km}$ EASE-Grid Day V005; Digital Media; National Snow and Ice Data Center: Boulder, CO, USA, 2006; updated daily.

19. Fowler, C. Polar Pathfinder Daily 25 km EASE-Grid Sea Ice Motion Vectors; Digital Media; National Snow and Ice Data Center: Boulder, CO, USA, 2003; updated 2008.

20. Armstrong, R.L.; Brodzik, M.J.; Knowles, K.; Savoie, M. Global Monthly EASE-Grid Snow Water Equivalent Climatology; Digital Media; National Snow and Ice Data Center: Boulder, CO, USA, 2007. 
21. Iliffe, J.; Lott, R. Datums and Map Projections for Remote Sensing, GIS and Surveying, 2nd ed.; Whittles Publishing: Scotland, UK, 2008.

22. Haran, T. HDF-EOS vs. GeoTIFF: GIS Problems When Projection and Datum Spheroids Are Different. Presentes at MODIS Science Team Meeting, Denver, CO, USA, 15-17 October 2008. Available online: ftp://sidads.colorado.edu/pub/ppp/conf_ppp/Haran/HDF-EOS_vs._GeoTIFF_GIS_Problems _when_Projection_and_Datum_Spheroids_are_Different.pdf （accessed on 27 November 2011).

23. Ritter, N.; Ruth, M. GeoTIFF Format Specification; GeoTIFF Revision 1.0; 1995. Available online: $\mathrm{ftp}$ //ftp.remotesensing.org/pub/geotiff/spec/geotiff.rtf (accessed on 27 November 2011).

24. Billingsley, B.; Haran, T. Using GeoTIFFs for Data Sharing: Limitations and Solutions. In Proceedings of AGU 2008 Fall Meeting, San Francisco, CA, USA, 15-19 December 2008; 89(53), Fall Meeting Suppl., Abstract IN23B-1078. Available online: ftp://sidads.colorado.edu/ pub/ppp/conf_ppp/Billingsley/Using_GeoTIFFs_for_Data_Sharing __Limitations_and_Solutions.pdf (accessed on 27 November 2011).

25. Meeus, J. The Earth's Globe. In Astronomical Algorithms; Chapter 10; Willmann-Bell: Richamond, VA, USA, 1991; pp. 77-82.

26. Snyder, J.P. Map Projections, A Working Manual; US Geological Survey Professional Paper 1395, Department of the Interior: Washington, DC, USA, 1987.

\section{Appendix A: EASE-Grid 2.0 Projection Formulae}

Projection formulae for EASE-Grid 2.0 as taken from [26] are as follows. (See Table 1 for WGS 84 ellipsoid parameters.)

\section{Definition. Let}

$a=$ equatorial radius

$b=$ polar radius

$e=$ eccentricity of the ellipsoid

$\phi_{0}=$ map reference latitude

$\lambda_{0}=$ map reference longitude

$\phi_{1}=$ latitude of true scale

$\phi=$ north latitude

$\lambda=$ longitude east of Greenwich

$x=$ distance (meters) to the right of the vertical line (Y axis) passing through the origin of the projection $y=$ distance (meters) above the horizontal line ( $X$ axis) passing through the origin of projection

For the cylindrical EASE-Grid 2.0, let:

$\phi_{0}=0.0^{\circ}$

$\lambda_{0}=0.0^{\circ}$

$\phi_{1}=30.0^{\circ}$

For the Northern azimuthal EASE-Grid 2.0, let: 
$\phi_{0}=90.0^{\circ}$

$\lambda_{0}=0.0^{\circ}$

For the Southern azimuthal EASE-Grid 2.0, let:

$\phi_{0}=-90.0^{\circ}$

$\lambda_{0}=0.0^{\circ}$

Definition (Cylindrical EASE-Grid 2.0 Forward Formulae ([26], p. 81)). Let

$$
\begin{aligned}
k_{0} & =\frac{\cos \phi_{1}}{\sqrt{1-e^{2} \sin ^{2} \phi_{1}}} \\
q(\phi) & =\left(1-e^{2}\right)\left[\frac{\sin \phi}{\left(1-e^{2} \sin ^{2} \phi\right)}-\frac{1}{2 e} \ln \left(\frac{1-e \sin \phi}{1+e \sin \phi}\right)\right]
\end{aligned}
$$

then

$$
\begin{aligned}
& x=a k_{0}\left(\lambda-\lambda_{0}\right) \\
& y=\frac{a q(\phi)}{2 k_{0}}
\end{aligned}
$$

Definition (Cylindrical EASE-Grid 2.0 Inverse Formulae ([26], pp. 82-83)). Use $k_{0}$ from Equation (1), use $q(\phi)$ from Equation (2), and let

$$
\begin{aligned}
q_{p} & =q\left(\phi=90^{\circ}\right) \\
\beta & =\arcsin \frac{2 y k_{0}}{a q_{p}}
\end{aligned}
$$

then $\phi$, (calculated by approximation), and $\lambda$ are

$$
\begin{aligned}
\phi= & \beta+\left(e^{2} / 3+31 e^{4} / 180+517 e^{6} / 5040\right) \sin 2 \beta \\
& +\left(23 e^{4} / 360+251 e^{6} / 3780\right) \sin 4 \beta \\
& +\left(761 e^{6} / 45360\right) \sin 6 \beta \\
\lambda= & \lambda_{0}+\frac{x}{a k_{0}}
\end{aligned}
$$

Definition (Azimuthal EASE-Grid 2.0 Forward Formulae ([26], pp. 187-188)). Use q( $\phi)$ from Equation (2), and let

$$
\begin{aligned}
q_{p}= & q\left(\phi=90^{\circ}\right) \\
\rho & = \begin{cases}0.0, & \text { if } q_{p}-q(\phi)=0 \\
a \sqrt{q_{p}-q(\phi)}, & \text { if North } \\
a \sqrt{q_{p}+q(\phi)}, & \text { if South }\end{cases}
\end{aligned}
$$


then

$$
\begin{aligned}
& x=\rho \sin \left(\lambda-\lambda_{0}\right) \\
& y= \begin{cases}-\rho \cos \left(\lambda-\lambda_{0}\right), & \text { if North } \\
\rho \cos \left(\lambda-\lambda_{0}\right), & \text { if South }\end{cases}
\end{aligned}
$$

Definition (Azimuthal EASE-Grid 2.0 Inverse Formulae ([26], p. 190)). Use $q(\phi)$ from Equation (2), and let

$$
\begin{aligned}
q_{p} & =q\left(\phi=90^{\circ}\right) \\
\rho & =\sqrt{x^{2}+y^{2}} \\
\beta & =\left\{\begin{array}{l}
\arcsin \left(1-\frac{\rho^{2}}{a^{2} q_{p}}\right), \text { if North } \\
-\arcsin \left(1-\frac{\rho^{2}}{a^{2} q_{p}}\right), \text { if South }
\end{array}\right.
\end{aligned}
$$

then $\phi$, (calculated by approximation), and $\lambda$ are

$$
\begin{aligned}
\phi= & \beta+\left(e^{2} / 3+31 e^{4} / 180+517 e^{6} / 5040\right) \sin 2 \beta \\
& +\left(23 e^{4} / 360+251 e^{6} / 3780\right) \sin 4 \beta \\
& +\left(761 e^{6} / 45360\right) \sin 6 \beta \\
\lambda= & \begin{cases}\lambda_{0}+\arctan \left(\frac{x}{-y}\right), & \text { if North } \\
\lambda_{0}+\arctan \left(\frac{x}{y}\right), & \text { if South }\end{cases}
\end{aligned}
$$

\section{Appendix B: Authalic Sphere Definition}

An authalic sphere is a sphere whose surface area is equal to that of some reference ellipsoid. The radius of the International 1924 Authalic Sphere is calculated from the International 1924 Ellipsoid using [26], Equation 3-13 on p. 16: Let

$$
q_{p}=1-\left[\frac{1-e^{2}}{2 e} \ln \left(\frac{1-e}{1+e}\right)\right]
$$

then

$$
R_{q}=a \sqrt{\frac{q_{p}}{2}}
$$

\section{Appendix C: EASE-Grid 2.0 Projection Parameters}

As funding allows, NSIDC is committed to supporting EASE-Grid 2.0. The authors plan to keep EASE-Grid Data pages at http://nsidc.org/data/ease/ updated with developments and changes related to the EASE-Grid 2.0 definition, including a description of the current state of support and known issues in popular software packages like GDAL tools and PROJ.4, HDFEOS, ENVI/IDL, ArcGIS and others. 
Table 2. PROJ.4 library EASE-Grid 2.0 projection parameters

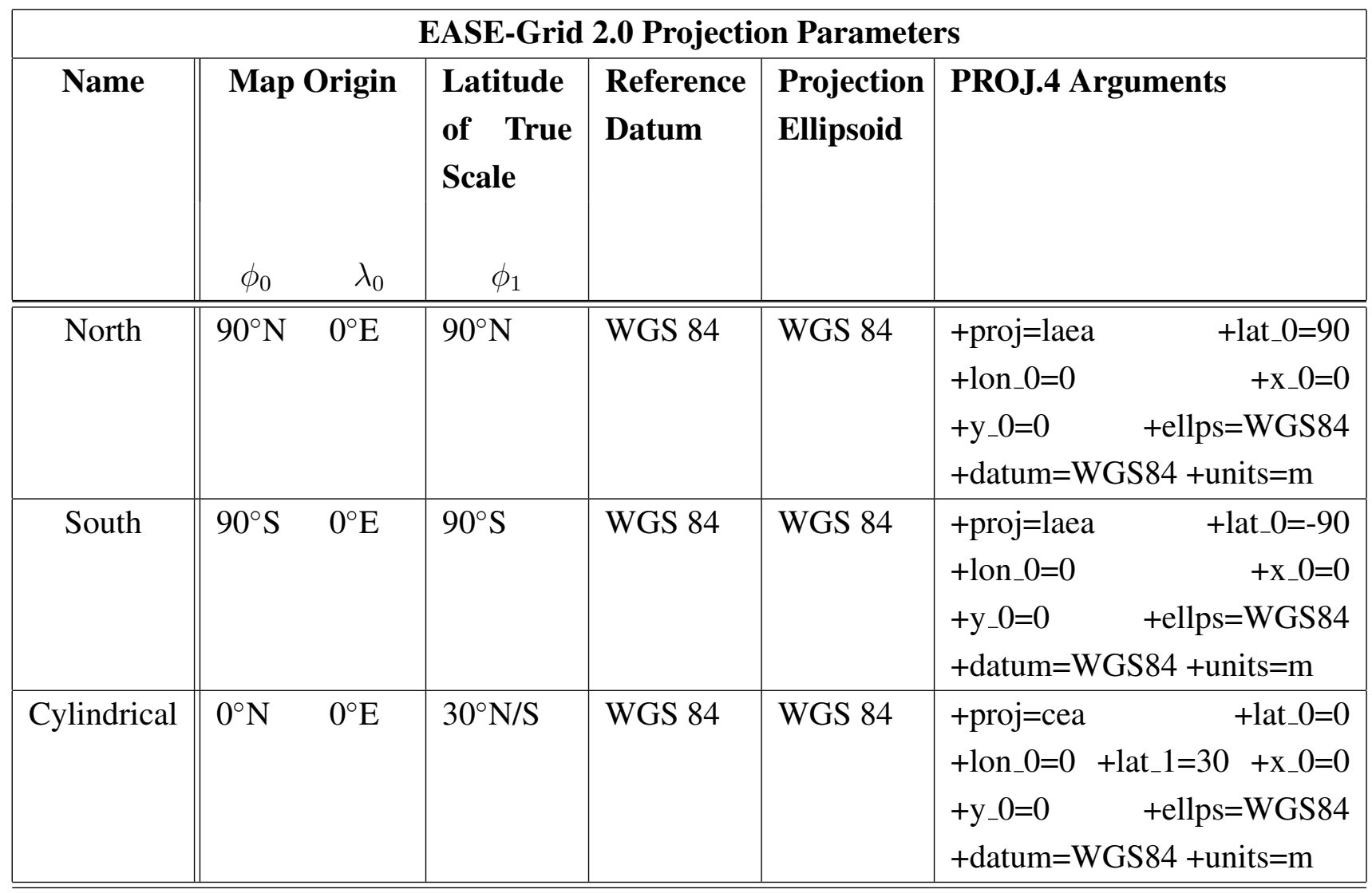

Table 2 lists projection parameters in the form of arguments to the popular PROJ.4 cartographic projections library (http://trac.osgeo.org/proj/). For grid parameters (dimensions, scale, nesting relationships) at specific scales (e.g., SMAP $9 \mathrm{~km}$ cylindrical EASE-Grid 2.0, etc.) please refer to EASE-Grid Data at http://nsidc.org/data/ease/.

(c) 2012 by the authors; licensee MDPI, Basel, Switzerland. This article is an open access article distributed under the terms and conditions of the Creative Commons Attribution license (http://creativecommons.org/licenses/by/3.0/). 